\title{
Dragging of inertial frames in the composed black-hole-ring system
}

\author{
Shahar Hod ${ }^{1,2, a}$ \\ ${ }^{1}$ The Ruppin Academic Center, 40250 Emeq Hefer, Israel \\ 2 The Hadassah Institute, 91010 Jerusalem, Israel
}

Received: 25 June 2015 / Accepted: 6 November 2015 / Published online: 19 November 2015

(C) The Author(s) 2015. This article is published with open access at Springerlink.com

\begin{abstract}
A well-established phenomenon in general relativity is the dragging of inertial frames by a spinning object. In particular, due to the dragging of inertial frames by a ring orbiting a central black hole, the angular velocity $\Omega_{\mathrm{H}}^{\mathrm{BH}-\text { ring }}$ of the black-hole horizon in the composed black-hole-ring system is no longer related to the black-hole angular momentum $J_{\mathrm{H}}$ by the simple Kerr-like (vacuum) relation $\Omega_{\mathrm{H}}^{\mathrm{Kerr}}\left(J_{\mathrm{H}}\right)=$ $J_{\mathrm{H}} / 2 M^{2} R_{\mathrm{H}}$ (here $M$ and $R_{\mathrm{H}}$ are the mass and horizon-radius of the black hole, respectively). Will has performed a perturbative treatment of the composed black-hole-ring system in the regime of slowly rotating black holes and found the explicit relation $\Omega_{\mathrm{H}}^{\mathrm{BH}-\text { ring }}\left(J_{\mathrm{H}}=0, J_{\mathrm{R}}, R\right)=2 J_{\mathrm{R}} / R^{3}$ for the angular velocity of a central black hole with zero angular momentum, where $J_{\mathrm{R}}$ and $R$ are respectively the angular momentum of the orbiting ring and its proper circumferential radius. Analyzing a sequence of black-hole-ring configurations with adiabatically varying (decreasing) circumferential radii, we show that the expression found by Will for $\Omega_{\mathrm{H}}^{\mathrm{BH}-\text { ring }}\left(J_{\mathrm{H}}=0, J_{\mathrm{R}}, R\right)$ implies a smooth transition of the central black-hole angular velocity from its asymptotic nearhorizon value $\Omega_{\mathrm{H}}^{\mathrm{BH}-\text { ring }}\left(J_{\mathrm{H}}=0, J_{\mathrm{R}}, R \rightarrow R_{\mathrm{H}}^{+}\right) \rightarrow 2 J_{\mathrm{R}} / R_{\mathrm{H}}^{3}$ (that is, just before the assimilation of the ring by the central black hole), to its final Kerr (vacuum) value $\Omega_{\mathrm{H}}^{\mathrm{Kerr}}\left(J_{\mathrm{H}}^{\text {new }}\right)=$ $J_{\mathrm{H}}^{\text {new }} / 2 M^{\text {new } 2} R_{\mathrm{H}}^{\text {new }}$ [that is, after the adiabatic assimilation of the ring by the central black hole. Here $J_{\mathrm{H}}^{\text {new }}=J_{\mathrm{R}}, M^{\text {new }}$, and $R_{\mathrm{H}}^{\text {new }}$ are the new parameters of the resulting Kerr (vacuum) black hole after it assimilated the orbiting ring]. We use this important observation in order to generalize the result of Will to the regime of black-hole-ring configurations in which the central black holes possess non-zero angular momenta. In particular, it is shown that the continuity argument (namely, the characteristic smooth evolution of the black-hole angular velocity during an adiabatic assimilation process of the
\end{abstract}

a e-mail: shaharhod@gmail.com ring into the central black hole) yields a concrete prediction for the angular-velocity/angular-momentum asymptotic functional relation $\Omega_{\mathrm{H}}^{\mathrm{BH}-\text { ring }}=\Omega_{\mathrm{H}}^{\mathrm{BH}-\text { ring }}\left(J_{\mathrm{H}}, J_{\mathrm{R}}, R \rightarrow R_{\mathrm{H}}^{+}\right)$ of generic (that is, with $J_{\mathrm{H}} \neq 0$ ) black-hole-ring configurations. Remarkably, we find the simple universal relation $\Delta \Omega_{\mathrm{H}} \equiv \Omega_{\mathrm{H}}^{\mathrm{BH}-\text { ring }}\left(J_{\mathrm{H}}, J_{\mathrm{R}}, R \rightarrow R_{\mathrm{H}}^{+}\right)-\Omega_{\mathrm{H}}^{\mathrm{Kerr}}\left(J_{\mathrm{H}}\right)=$ $J_{\mathrm{R}} / 4 M^{3}$ for the asymptotic deviation of the black-hole angular velocity in the composed black-hole-ring system from the corresponding angular velocity of the unperturbed (vacuum) Kerr black hole with the same angular momentum.

\section{Introduction}

The gravitational two-body problem has attracted much attention over the years from both physicists and mathematicians. In particular, it is highly important to explore the physics of a central black hole surrounded by an orbiting ring: it is expected that this composed two-body system may be formed as an intermediate stage in the gravitational collapse of a compact spinning star to form a black hole [1-3]. Likewise, the coalescence of two compact objects may produce a composed black-hole-ring system [1-3]. In addition to these astrophysical motivations, it is highly interesting to explore the composed black-hole-ring system in order to understand how an exterior matter configuration affects the physical properties of central black holes [1-5].

The general-relativistic problem of a slowly spinning black hole surrounded by a thin orbiting ring was studied perturbatively by Will $[4,5]$ (see also [6-8]). It was shown in [4] that, due to the well-known phenomenon of dragging of inertial frames by the orbiting ring, the angular velocity $\Omega_{\mathrm{H}}^{\mathrm{BH}-\text { ring } 1}$ of the central black hole in the composed black-

\footnotetext{
1 We use the symbol $\Omega_{\mathrm{H}}^{\mathrm{BH}-\mathrm{ring}}$ to denote the angular velocity of the black-hole horizon in the composed black-hole-ring system.
} 
hole-ring system is no longer related to the black-hole angular momentum $J_{\mathrm{H}}$ by the simple Kerr-like (vacuum) relation

$\Omega_{\mathrm{H}}^{\mathrm{Kerr}}\left(J_{\mathrm{H}}\right)=\frac{J_{\mathrm{H}}}{2 M^{2} R_{\mathrm{H}}}$.

[Here $M$ and $R_{\mathrm{H}}=M+\left(M^{2}-J_{\mathrm{H}}^{2} / M^{2}\right)^{1 / 2}$ are the mass and horizon-radius of the black hole, respectively]. In particular, Will [4] has demonstrated explicitly that, in the composed black-hole-ring system, one can have a central black hole with zero angular momentum but with a non-zero angular velocity $^{2}$

$\Omega_{\mathrm{H}}^{\mathrm{BH}-\text { ring }}\left(J_{\mathrm{H}}=0, J_{\mathrm{R}}, R\right)=\frac{2 J_{\mathrm{R}}}{R^{3}}$,

where $J_{\mathrm{R}}$ and $R$ are, respectively, the angular momentum of the orbiting ring and its proper circumferential radius. To the best of our knowledge, no exact (analytical) calculations of the frame-dragging effect have been performed for generic black-hole-ring configurations (that is, for the case of central black holes with non-negligible angular momenta).

\section{The continuous (smooth) behavior of the black-hole angular velocity}

The main goal of the present paper is to generalize the result (2) of [4] to the regime of composed black-hole-ring configurations in which the central black holes possess non-zero angular momenta. In particular, we shall use a simple continuity argument in order to provide a concrete analytical prediction for the angular-velocity/angular-momentum asymptotic functional relation $\Omega_{\mathrm{H}}^{\mathrm{BH}-\text { ring }}=\Omega_{\mathrm{H}}^{\mathrm{BH}-\text { ring }}\left(J_{\mathrm{H}}, J_{\mathrm{R}}, R \rightarrow\right.$ $R_{\mathrm{H}}^{+}$) of generic (that is, with $J_{\mathrm{H}} \neq 0$ ) central black holes in the composed black-hole-ring system.

Our approach here is based on a continuity argument for the behavior of the black-hole angular velocity in an adiabatic process in which the orbiting ring is assimilated (adiabatically lowered) into the central black hole. In order to demonstrate the idea, we shall first analyze the analytical relation (2) of [4] for the angular velocity of a zero angular momentum $\left(J_{\mathrm{H}}=0\right)$ central black hole.

Let us first consider a sequence of black-hole-ring configurations with adiabatically varying (decreasing) circumferential radii. Inspection of Eq. (2) reveals that, for a given value of the ring angular momentum $J_{\mathrm{R}}$, the central blackhole angular velocity increases as the ring approaches the

${ }^{2}$ This results is valid in the perturbative regime $J_{\mathrm{R}} / R^{2} \ll 1$. There are also subleading correction terms of order $O\left(J_{\mathrm{R}}^{2} / R^{5}\right)$ on the r.h.s. of this relation. black-hole horizon (that is, as $R$ decreases). In particular, taking the near-horizon limit $R \rightarrow R_{\mathrm{H}}^{+}$in (2), one finds ${ }^{3}$

$\Omega_{\mathrm{H}}^{\mathrm{BH}-\text { ring }}\left(J_{\mathrm{H}}=0, J_{\mathrm{R}}, R \rightarrow R_{\mathrm{H}}^{+}\right) \rightarrow \frac{J_{\mathrm{R}}}{4 M^{3}}$

for the angular velocity of the central black hole just before it assimilates the ring.

Let us now calculate the new angular velocity $\Omega_{\mathrm{H}}^{\mathrm{Kerr}}\left(J_{\mathrm{H}}^{\text {new }}\right)$ of the resulting Kerr (vacuum) black hole after it absorbed the ring. The adiabatic assimilation of the rotating ring by the central black hole produces the following changes in the black-hole physical parameters:

$M \rightarrow M^{\text {new }}=M+\mathcal{E}_{\mathrm{R}}$ and $J_{\mathrm{H}}=0 \rightarrow J_{\mathrm{H}}^{\text {new }}=J_{\mathrm{R}}$,

where the energy $\mathcal{E}_{\mathrm{R}}$ of the rotating ring at the absorption point $R=R_{\mathrm{H}}$ is given by [9] $]^{4,5,6}$

$\mathcal{E}_{\mathrm{R}}=\frac{J_{\mathrm{H}}}{2 M^{2} R_{\mathrm{H}}} \cdot J_{\mathrm{R}} \rightarrow 0$ for $J_{\mathrm{H}}=0$.

Substituting (4) and (5) into (1), one finds (see footnote 3)

$\Omega_{\mathrm{H}}^{\text {Kerr }}\left(J_{\mathrm{H}}^{\text {new }}=J_{\mathrm{R}}\right)=\frac{J_{\mathrm{R}}}{4 M^{3}}$

for the angular velocity of the final (vacuum) Kerr black hole. $^{7}$

Comparing the near-horizon asymptotic $\left(R \rightarrow R_{\mathrm{H}}^{+}\right)$ expression (3) for the angular velocity of the central black hole in the composed black-hole-ring system just before the assimilation of the ring, with Eq. (6) for the angular velocity of the resulting Kerr (vacuum) black hole after it assimilated the ring, one concludes that the black hole is characterized by a smooth (continuous) evolution of its angular velocity during the adiabatic assimilation process. That is,

$\Omega_{\mathrm{H}}^{\mathrm{Kerr}}\left(J_{\mathrm{H}}^{\text {new }}=J_{\mathrm{R}}\right)=\Omega_{\mathrm{H}}^{\mathrm{BH}-\text { ring }}\left(J_{\mathrm{H}}=0, J_{\mathrm{R}}, R \rightarrow R_{\mathrm{H}}^{+}\right)$.

\footnotetext{
${ }^{3}$ Here we have used the leading-order relation $R_{\mathrm{H}}=2 M$ for the horizon-radius of the zero angular-momentum black hole.

${ }^{4}$ We consider here an adiabatic assimilation process of the ring into the central black hole. In this idealized scenario the assimilated ring has a zero radial momentum at the point of capture.

5 It is worth emphasizing that the mass-energy of the ring as measured by asymptotic observers, $\sqrt{g_{00}} \mu$, is completely red-shifted $\left(\sqrt{g_{00}}=0\right)$ at the absorption point (at the black-hole horizon).

6 This results is valid in the perturbative regime $J_{\mathrm{R}} / R^{2} \ll 1$. There are also small (subleading) correction terms of order $O\left(J_{\mathrm{R}}^{2} / R^{3}, \mu^{2} / R\right)$ on the r.h.s. of this relation.

7 Note that the black-hole surface area $8 \pi M R_{\mathrm{H}}$ plays the role of an adiabatic invariant (see, Bekenstein [10]). It therefore acquires small (subleading) $O\left(J_{\mathrm{R}}^{2} / M^{2}, \mu^{2}\right)$ corrections, which are only second-order in the ring parameters.
} 


\section{The angular-velocity/angular-momentum relation for generic black-hole-ring configurations}

In the present section we shall analyze the angular-velocity/ angular-momentum asymptotic functional relation $\Omega_{\mathrm{H}}^{\mathrm{BH}-\text { ring }}=\Omega_{\mathrm{H}}^{\mathrm{BH}-\text { ring }}\left(J_{\mathrm{H}}, J_{\mathrm{R}}, R \rightarrow R_{\mathrm{H}}^{+}\right)$of generic (that is, with $J_{\mathrm{H}} \neq 0$ ) central black holes in the composed blackhole-ring system. To that end, we shall use the characteristic continuity relation ${ }^{8}$

$\Omega_{\mathrm{H}}^{\mathrm{Kerr}}\left(J_{\mathrm{H}}^{\text {new }}=J_{\mathrm{H}}+J_{\mathrm{R}}\right)=\Omega_{\mathrm{H}}^{\mathrm{BH}-\text {-ring }}\left(J_{\mathrm{H}}, J_{\mathrm{R}}, R \rightarrow R_{\mathrm{H}}^{+}\right)$

for the evolution of the black-hole angular velocity during an adiabatic assimilation process of the orbiting ring by the central black hole.

We consider a composed black-hole-ring system which is characterized by the physical parameters $J_{\mathrm{H}}, J_{\mathrm{R}}$, and $R$. The adiabatic absorption of the ring by the central black hole produces a final Kerr (vacuum) black hole with the following parameters:

$M \rightarrow M^{\text {new }}=M+\mathcal{E}_{\mathrm{R}}$ and $J_{\mathrm{H}} \rightarrow J_{\mathrm{H}}^{\text {new }}=J_{\mathrm{H}}+J_{\mathrm{R}}$,

where the energy $\mathcal{E}_{\mathrm{R}}$ of the ring at the absorption point $R=$ $R_{\mathrm{H}}$ is given by [9] (see footnote 4-6)

$\mathcal{E}_{\mathrm{R}}=\frac{J_{\mathrm{H}}}{2 M^{2} R_{\mathrm{H}}} \cdot J_{\mathrm{R}}$

Substituting (9) and (10) into (1), one finds

$\Omega_{\mathrm{H}}^{\mathrm{Kerr}}\left(J_{\mathrm{H}}^{\text {new }}=J_{\mathrm{H}}+J_{\mathrm{R}}\right)=\frac{J_{\mathrm{H}}}{2 M^{2} R_{\mathrm{H}}}+\frac{J_{\mathrm{R}}}{4 M^{3}}$

for the angular velocity of the final (vacuum) Kerr black hole (see footnote 7).

Taking cognizance of Eqs. (1) and (11), and using the continuity argument (8) for the evolution of the black-hole angular velocity during the adiabatic assimilation process of the ring into the central black hole, one finds the characteristic angular-velocity/angular-momentum asymptotic relation

$\Omega_{\mathrm{H}}^{\mathrm{BH}-\text { ring }}\left(J_{\mathrm{H}}, J_{\mathrm{R}}, R \rightarrow R_{\mathrm{H}}^{+}\right)=\Omega_{\mathrm{H}}^{\mathrm{Kerr}}\left(J_{\mathrm{H}}\right)+\frac{J_{\mathrm{R}}}{4 M^{3}}$

for a central black hole of angular momentum $J_{\mathrm{H}}$ in the composed black-hole-ring system [here $\Omega_{\mathrm{H}}^{\mathrm{Kerr}}\left(J_{\mathrm{H}}\right)$, as given by

\footnotetext{
${ }^{8}$ Note that the r.h.s. of (8) refers to the angular velocity of the central black hole in the composed black-hole-ring system in the near-horizon limit $R \rightarrow R_{\mathrm{H}}^{+}$(that is, just before the assimilation of the ring by the black hole), whereas the 1.h.s. of (8) refers to the angular velocity of the final Kerr (vacuum) black hole (that is, after it assimilated the ring).
}

(1), is the angular velocity of a (vacuum) Kerr black hole with the same angular momentum].

\section{Summary and discussion}

The composed black-hole-ring system is one of the most fundamental problems in general relativity and astrophysics [13]. This two-body system is characterized by one of the most intriguing phenomena in general relativity, namely the dragging of inertial frames. In a very interesting work, Will $[4,5]$ studied this composed system perturbatively in the regime of slowly spinning central black holes. It was shown in $[4,5]$ that the effect of dragging of inertial frames by the orbiting ring yields a non-trivial angular-velocity/angular-momentum relation for the central black hole. In particular, Will $[4,5]$ found the non-zero angular velocity (2) for a central black hole of zero angular momentum $\left(J_{\mathrm{H}}=0\right)$ in the composed black-hole-ring system.

To the best of our knowledge, in the physical literature there are no available analytical results for the framedragging effect in generic black-hole-ring configurations (that is, for central black holes with non-negligible angular momenta).

The main goal of the present paper was to generalize the result (2) of Will [4,5] to the regime of composed black-holering configurations in which the central black holes possess non-zero angular momenta.

In particular, we have explored the angular-velocity/ angular-momentum asymptotic functional relation $\Omega_{\mathrm{H}}^{\mathrm{BH}-\text { ring }}=\Omega_{\mathrm{H}}^{\mathrm{BH}-\text { ring }}\left(J_{\mathrm{H}}, J_{\mathrm{R}}, R \rightarrow R_{\mathrm{H}}^{+}\right)$of generic blackhole-ring configurations. To that end, we have used a continuity argument ${ }^{9}$ for the evolution of the black-hole angular velocity during a physical process in which the orbiting ring is adiabatically lowered into the central black hole. This continuity argument [see Eqs. (7), (8)] yields the nontrivial (non-Kerr-like) angular-velocity/angular-momentum asymptotic functional relation (12) for generic (that is, with $J_{\mathrm{H}} \neq 0$ ) central black holes in the composed black-hole-ring system.

Remarkably, our result (12) for the angular velocity of the perturbed central black hole implies the simple universal $^{10}$ relation

$\Delta \Omega_{\mathrm{H}}\left(R \rightarrow R_{\mathrm{H}}^{+}\right)=\frac{J_{\mathrm{R}}}{4 M^{3}}$

\footnotetext{
9 That is, a smooth functional behavior [see Eqs. (7) and (8)] of the central black-hole angular velocity in an adiabatic assimilation process of the orbiting ring into the central black hole.

${ }^{10}$ Note that the relation (13) for $\Delta \Omega_{\mathrm{H}}$ is universal in the sense that it is independent of the black-hole angular momentum $J_{\mathrm{H}}$.
} 
where $\Delta \Omega_{\mathrm{H}}\left(R \rightarrow R_{\mathrm{H}}^{+}\right) \equiv \Omega_{\mathrm{H}}^{\mathrm{BH}-\text { ring }}\left(J_{\mathrm{H}}, J_{\mathrm{R}}, R \rightarrow R_{\mathrm{H}}^{+}\right)-$ $\Omega_{\mathrm{H}}^{\mathrm{Kerr}}\left(J_{\mathrm{H}}\right)$ is the asymptotic deviation of the black-hole angular velocity $\Omega_{\mathrm{H}}^{\mathrm{BH}-\text { ring }}\left(J_{\mathrm{H}}\right)$ in the composed black-hole-ring system from the corresponding angular velocity $\Omega_{\mathrm{H}}^{\mathrm{Kerr}}\left(J_{\mathrm{H}}\right)$ [see Eq. (1)] of the unperturbed (vacuum) Kerr black hole with the same angular momentum $J_{\mathrm{H}}$. It is worth emphasizing that the asymptotic relation (13) for $\Delta \Omega_{\mathrm{H}}$ is universal in the sense that it is independent of the black-hole angular momentum $J_{\mathrm{H}}$.

Finally, we would like to end this paper we a conjecture. In particular, we would like to suggest a simple (and compact) formula which generalizes the asymptotic nearhorizon result (13) to generic values of the ring radius $R$. To that end, we note that the simplest ${ }^{11}$ functional relation $\Delta \Omega_{\mathrm{H}}=\Delta \Omega_{\mathrm{H}}(R)$, which reduces to (2) in the zero angularmomentum $J_{\mathrm{H}} \rightarrow 0$ limit, ${ }^{12}$ and to (13) in the asymptotic near-horizon $R \rightarrow R_{\mathrm{H}}$ limit, is given by ${ }^{13}$

$\Delta \Omega_{\mathrm{H}}(R)=\frac{J_{\mathrm{R}}}{4 M^{3}} \cdot\left(\frac{R_{\mathrm{H}}}{R}\right)^{3}$.

It would be highly interesting to test the validity of our conjectured relation (14) with full non-linear ${ }^{14}$ numerical computations for generic (that is, with $J_{\mathrm{H}} \neq 0$ ) black-hole-ring configurations.
Acknowledgments This research is supported by the Carmel Science Foundation. I thank Yael Oren, Arbel M. Ongo, Ayelet B. Lata, and Alona B. Tea for stimulating discussions.

Open Access This article is distributed under the terms of the Creative Commons Attribution 4.0 International License (http://creativecomm ons.org/licenses/by/4.0/), which permits unrestricted use, distribution, and reproduction in any medium, provided you give appropriate credit to the original author(s) and the source, provide a link to the Creative Commons license, and indicate if changes were made. Funded by $\mathrm{SCOAP}^{3}$.

\section{References}

1. M. Ansorg, D. Petroff, Phys. Rev. D 72, 024019 (2005)

2. N.L. Shapiro, Astrophys. J. 444, 306 (1995)

3. M. Shibata, K. Taniguchi, K. Uryu, Phys. Rev. D 68, 084020 (2003)

4. C.M. Will, Astrophys. J. 191, 521 (1974)

5. C.M. Will, Astrophys. J. 196, 41 (1975)

6. S. Hod, Phys. Rev. D 87, 024036 (2013). arXiv:1311.1281

7. S. Hod, Phys. Lett. B 726, 533 (2013). arXiv:1312.4969

8. S. Hod, Eur. Phys. J. C 74, 2840 (2014). arXiv:1404.1566

9. B. Carter, Phys. Rev. 174, 1559 (1968)

10. J.D. Bekenstein, in Black Holes: Classical Properties, Thermodynamics and Heuristic Quantization. ed. by M. Novello. Cosmology and Gravitation (Atlantisciences, France, 2000), pp. 1-85. arXiv:gr-qc/9808028

\footnotetext{
11 It should be emphasized that the conjectured relation (14) is not unique. For example, the more complicated expression $\Delta \Omega_{\mathrm{H}}(R)=$ $\frac{R R_{\mathrm{H}} J_{\mathrm{R}}}{M\left(R^{2}+J_{\mathrm{H}}^{2} / M^{2}\right)^{2}}$ also reduces to (2) in the zero angular-momentum $J_{\mathrm{H}} \rightarrow$ 0 limit (which corresponds to $R_{\mathrm{H}} \rightarrow 2 M$ ), and to (13) in the asymptotic near-horizon $R \rightarrow R_{\mathrm{H}}$ limit.

12 Note that this limit corresponds to $R_{\mathrm{H}} \rightarrow 2 M$.

13 Note that $R_{\mathrm{H}} \rightarrow 2 M$ in the zero angular-momentum $J_{\mathrm{H}} \rightarrow 0$ limit, in which case (14) reduces to (2). Likewise, $R \rightarrow R_{\mathrm{H}}$ in the near-horizon limit, in which case (14) reduces to (13).

${ }^{14}$ It is worth emphasizing again that our analysis is valid in the perturbative regime $J_{\mathrm{R}} / R^{2} \ll 1$.
} 